\begin{tabular}{|c|l|}
\hline Title & Axial elongation of water droplet by application of electric field for bio-chemical reaction system \\
\hline Author(s) & Hino, T.; Hyashishita, E.; Y amauchi, Y.; Hirohata, Y.; Nishikawa, M. \\
\hline Citation & $\begin{array}{l}\text { Physica Scripta, T118, 193 195 } \\
\text { https://doi.org/10.1238/Physica.T Topical.118a00193 }\end{array}$ \\
\hline Issue Date & 2005 \\
\hline Doc URL & http://hdl.handle.net/2115/13699 \\
\hline Type & article \\
\hline File Information & paper\#1.pdf \\
\hline
\end{tabular}

Instructions for use 
Paper code: $68 . \mathrm{htm}$, Session 4

\title{
Axial Elongation of Water Droplet by Application of Electric Field for Bio-chemical Reaction System
}

\author{
T. Hino ${ }^{1}$, E. Hyashishita ${ }^{1}$, Y. Yamauchi1, Y. Hirohata ${ }^{1}$, M. Nishikawa ${ }^{2}$
}

${ }^{1}$ Laboratory for Plasma Physics and Engineering, Hokkaido University, Kita-13, Nishi-8, Kita-ku, Sapporo, 060-8628 Japan, ${ }^{2}$ Science Solutions Inc., Naka-machi 2-21-7, Meguro-ku, Tokyo, 153-0065 Japan

$$
\text { tomhino@qe.eng.hokudai.ac.jp }
$$

PACs Number: 52.30.-q, 52.25.Mg, 52.30.Cv

Keywords: Water, Elongation, Electric Field, Electric Energy

\begin{abstract}
In bio-micro devices, numerous functions of liquid flow such as elongation, expansion and transportation are required. Electro-wetting is one of the methods to control the liquid flow. In the present study, a water droplet was elongated in a channel based upon the electro-wetting, and the effect of electrode diameter and shape of electrode immersed in a water droplet on the elongation was examined. The elongation increased as a diameter of electrode, and significantly increased when the electrode with a T-shape was employed. The electric field at the edge of water droplet is kept relatively large when the rod electrode with a large diameter or the T-shape electrode is employed. The present result can be explained by this reason, the change of local electric energy at the edge of the droplet.
\end{abstract}

\section{Introduction}

In a micro electric mechanical system, MEMS, and small chemical reaction device, one of key issues is to precisely control the liquid flow in a micro size channel and to drive the liquid into a reaction chamber or cell. For this purpose, numerous motions of liquid flow such as transport speed and quantity, elongation, expansion, and stopping have to be controlled. As one of the control methods, an electric field has been applied for liquid to cause electro-wetting [1-4] or electro-osmotic flow $[5,6]$. In the electro-wetting, a liquid is placed on a dielectric thin film and electric field is applied between the liquid and the dielectric film. Then the liquid expands since the shape kept by a surface tension is destroyed by the electric energy. This phenomenon is well known as Lippmann effect. If electrodes are arrayed along the liquid flow channel, a liquid flow continuously moves through the channel $[3,4]$. It is also known that the expansion or the elongation of the water droplet saturates even if the electric field is increased [1, 2]. Thus, it is required to examine the limit of liquid expansion or elongation for a system with a single electrode 
configuration, and to develop the method to extend the limit.

In the present study, first, the expansion of a water droplet was measured by changing the electric field and a saturation of the expansion was confirmed. In order to enhance the expansion or elongation, the diameter of electrode immersed in the liquid and the shape of electrode were changed, and then the effect of electrode shape on the expansion or elongation was investigated.

\section{Experiments and Results}

A simple experiment of electro-wetting was conducted in the apparatus shown in Fig. 1. A water droplet was placed on the dielectric material, polyethylene sheet with a thickness of $30 \mu \mathrm{m}$. The volume of water droplet was $0.063 \mathrm{~cm}^{3}$. As the electrode immersed in the liquid, platinum rod with a diameter of $0.3 \mathrm{~mm}$ was used. Aluminum sheet was employed as the counter electrode. The change of the contact angle was observed by using a digital camera, Nikon E950. The voltage of applied electric field was increased from 0 to $500 \mathrm{~V}$ and the change of contact angle was observed.

Figures 2 (a) and (b) show the change of contact angle and changes of spread and height of droplet, respectively. The initial contact angle was $72.5^{\circ}$ and the contact angle decreased to $66.5^{\circ}$. The initial diameter of water droplet was $6.39 \mathrm{~mm}$ and the distance of radial spread was as high as $0.30 \mathrm{~mm}$. The initial height was $2.59 \mathrm{~mm}$ and the reduction of height was as high as $0.21 \mathrm{~mm}$. The expansion of water droplet continuously increased for the voltage lower than approximately $200 \mathrm{~V}$ and saturated for the range higher than $200 \mathrm{~V}$.

In order to enhance the expansion of water droplet, the diameter of platinum electrode was changed in the apparatus where the water droplet was placed in a channel with a radius of $\mathrm{a}=2 \mathrm{~mm}$ as shown in Fig. 3 (a). In this configuration, the polyethylene film and aluminum foil similar to the case of an apparatus shown Fig.1 was used as the dielectric material and counter electrode, respectively. The size of the rod diameter was taken 0.12, 0.3 and $0.5 \mathrm{~mm}$. As shown in Fig. 3 (b), the spread in the axial direction was expressed as an elongation ratio, $\Delta \mathrm{L} / \mathrm{a}$, where $\mathrm{L}$ and $\Delta \mathrm{L}$ are initial axial length and elongated length of water droplet, respectively. The volume of water droplet was $0.125 \mathrm{~cm}^{3}$ and the initial axial length $\mathrm{L}$ was in the range from 14.5 to $16.5 \mathrm{~mm}$. Figure 4 (a) and (b) show the elongation ratio versus applied voltage and elongation ratio versus electrode diameter, respectively. When the diameter was $0.12 \mathrm{~mm}$, the spread took place at the voltage $150 \mathrm{~V}$ and the maximum elongation ratio was observed to be small, 0.25 . In opposition, the maximum elongation ratio became large, 0.4 , when the diameter was $0.5 \mathrm{~mm}$, although the voltage required for the initiation of spread was large, $250 \mathrm{~V}$. These results suggest that the axial elongation can be enhanced by increase of electrode diameter.

It is known that the electro-wetting occurs when the electric energy exceeds the surface tension energy. The above experimental results indicate that the electric energy per unit volume at the edge of water droplet decreases when the spread increases, and the axial elongation saturates. The elongation or expansion may be able to be increased if the local electric energy at the edge of water droplet is taken relatively high even when the spread takes place. Thus, the use of rod electrode 
with a large diameter increased the axial elongation.

In order to more enhance the axial elongation, the electrode with a shape of figure $\mathrm{T}$ was employed and the horizontal part of T-shape electrode was immersed in the droplet as shown in Fig. 5. The volume of water droplet was $0.187 \mathrm{~cm}^{3}$ and the corresponding initial length was in the range from 19 to $21 \mathrm{~mm}$. The horizontal length of T-shape electrode, l, was taken 5, 10 and $15 \mathrm{~mm}$.

Figure 6 (a) and (b) show the elongation ratio versus applied voltage and elongation ratio versus horizontal length of electrode, respectively. As the increase of horizontal length, the elongation ratio significantly increased, compared with the case of rod electrode. When the horizontal length was taken longer than the axial length of droplet, no elongation occurred. This reason is discussed in the next session. The present results show that the elongation or spread can be enhanced if the reduction of electric energy at the edge of water droplet is suppressed by changing relative length of electrode to droplet.

\section{Discussion and Summary}

Several series of experiment on expansion or elongation of water droplet based upon electrowetting were conducted. The use of electrode with a large diameter was observed to be useful to lengthen the spread width. For the axial elongation of water droplet in a channel, the use of T-shape electrode significantly increased the axial spread width. The enhancement of spread occurs due to the change of spatial distribution of applied electric field. In the followings, the profile of electric field is discussed.

In the case that the rod electrode is employed, the applied electric field between the dielectric polyethylene film and water droplet changes according to the diameter of rod electrode as illustrated in Fig. 7. If the diameter of electrode is large, the reduction of electric field at the edge becomes smaller even if the spread takes place, and this is a possible reason that the use of electrode with a large diameter can expand the spread width. If the T-shape electrode is employed as shown in Fig. 8 (a), the electric field at the edge can be taken relatively higher. Thus, the elongation of water droplet placed on a channel can be significantly lengthened. In the case that the horizontal length exceeds the axial length of droplet, the polarized part of water droplet in the vicinity of electrode attaches the electrode and then the elongation does not occur as shown in Fig. 8 (b). Therefore, the present experimental results can be explained by the spatial distribution of electric field at the edge of water droplet.

In a small micro chemical device, one of key functions is to expand a liquid flow in a tube and to drive the flow into a reaction cells by a remote control such as application of electric field. The degree of the expansion or elongation has to be precisely controlled. For this purpose, use of electrode with different size and/or use of $\mathrm{T}$-shape electrode may be valuable. Instead of T-shape electrode, the elongation in two channels can become possible if U-shape electrode is employed. 


\section{Acknowledgement}

The authors acknowledge to Prof. and Dr. T. Ohkawa, Toyo Technologies Inc., USA, for valuable comments and discussions.

\section{References}

[1] M. Vallet, M. Vallade and B. Berge, Eur. Phys., B11 (1999) 583.

[2] M. Vallet, B. Berge and L. Vovelle, Polymer, 37 (1996) 2465.

[3] J. Lee and C. J. Kim, "Liquid Micrometer Driven by Continuous Electrowetting", Proc. IEEE MEMS '98, Heidelberg, Germany, pp. 538-543, 1998.

[4] J. Lee and C. J. Kim, "Microactuation by Continuous Electrowetting Phenomenon and Deep RIE Process", Proc. MEMS, ASME IMECE '98, CA, USA, pp.475-480, 1998.

[5] C.-H. Chan and J. G. Santiago, J. Microelectromechanical Systems, 11 (2002) 672.

[6] D. J. Harrison, K. Fluri, K. Seiler, Z. Fan and C. S. Effenhauser, Science, 261 (1993) 895. 
DC power supply

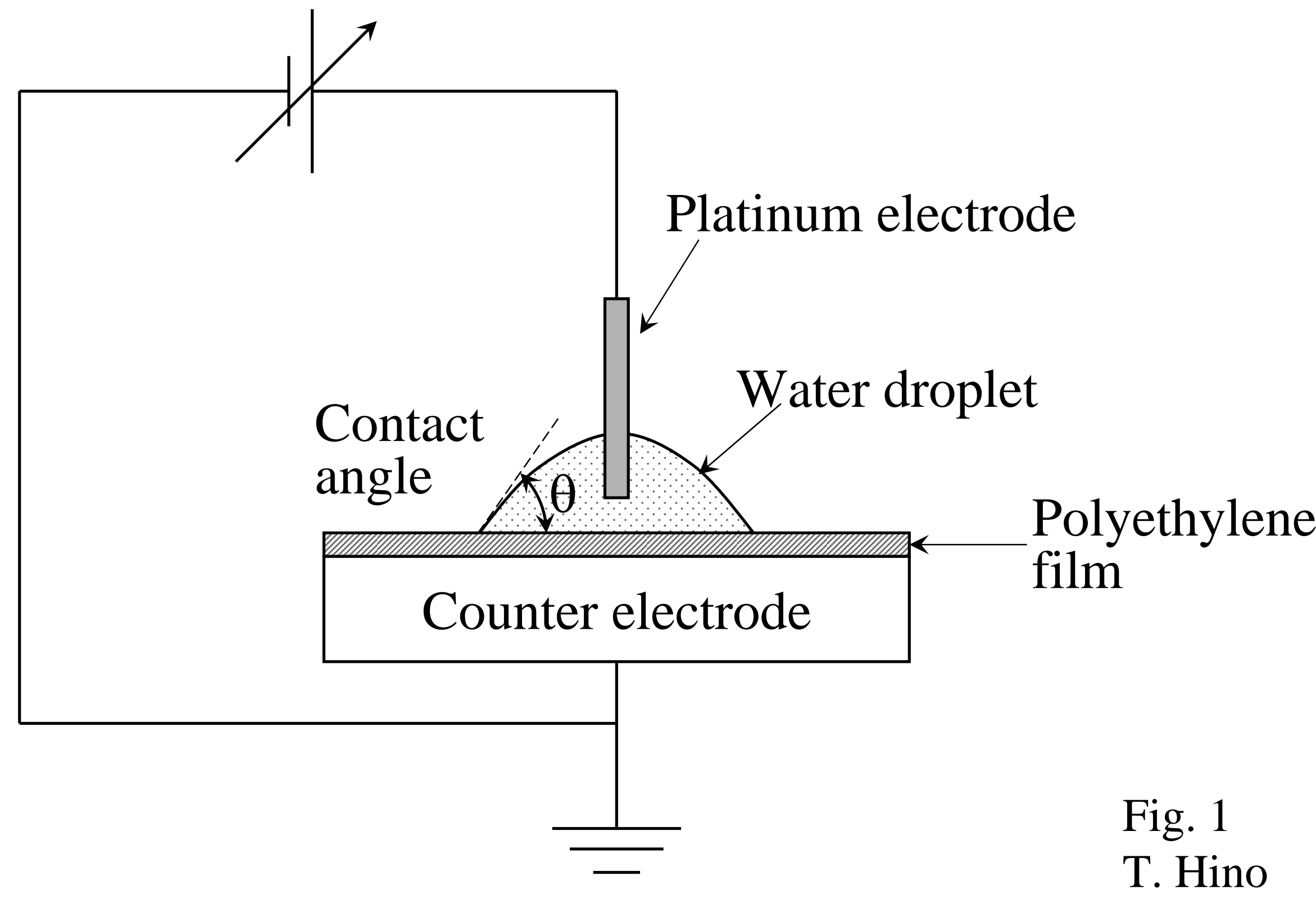


(a)

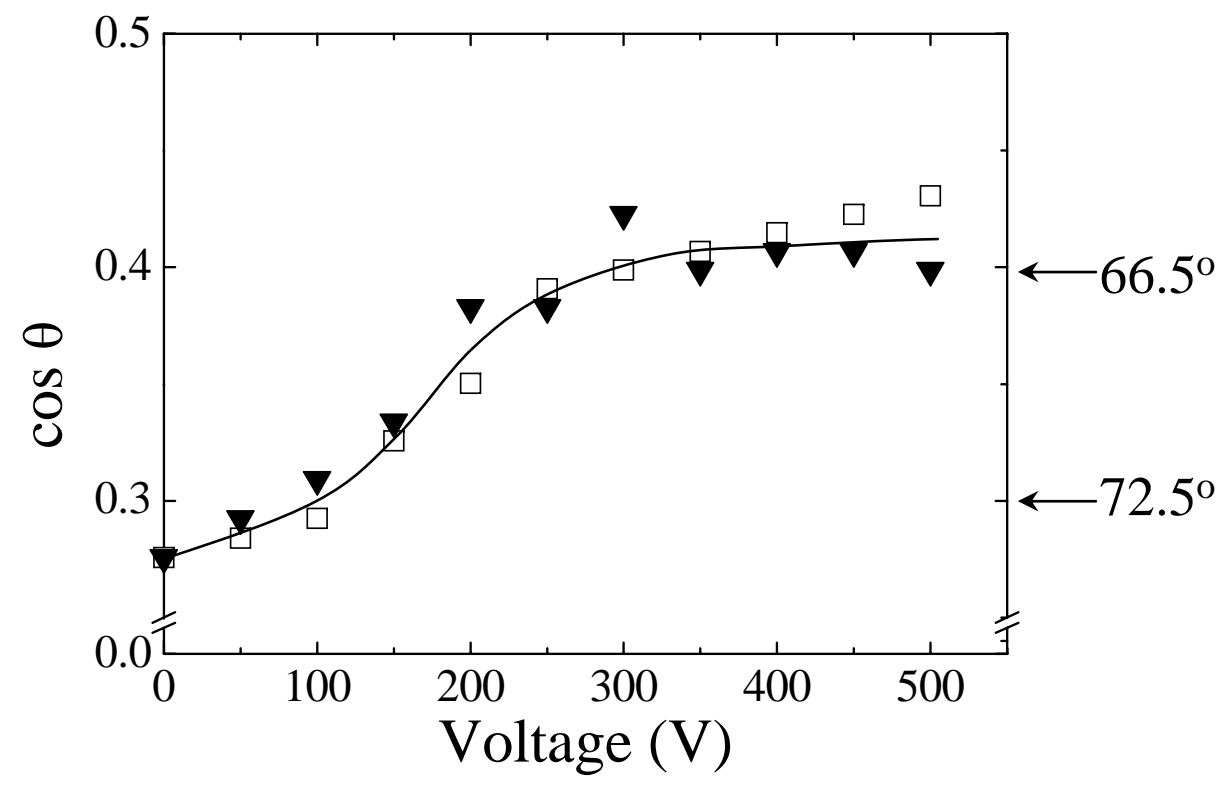

(b)

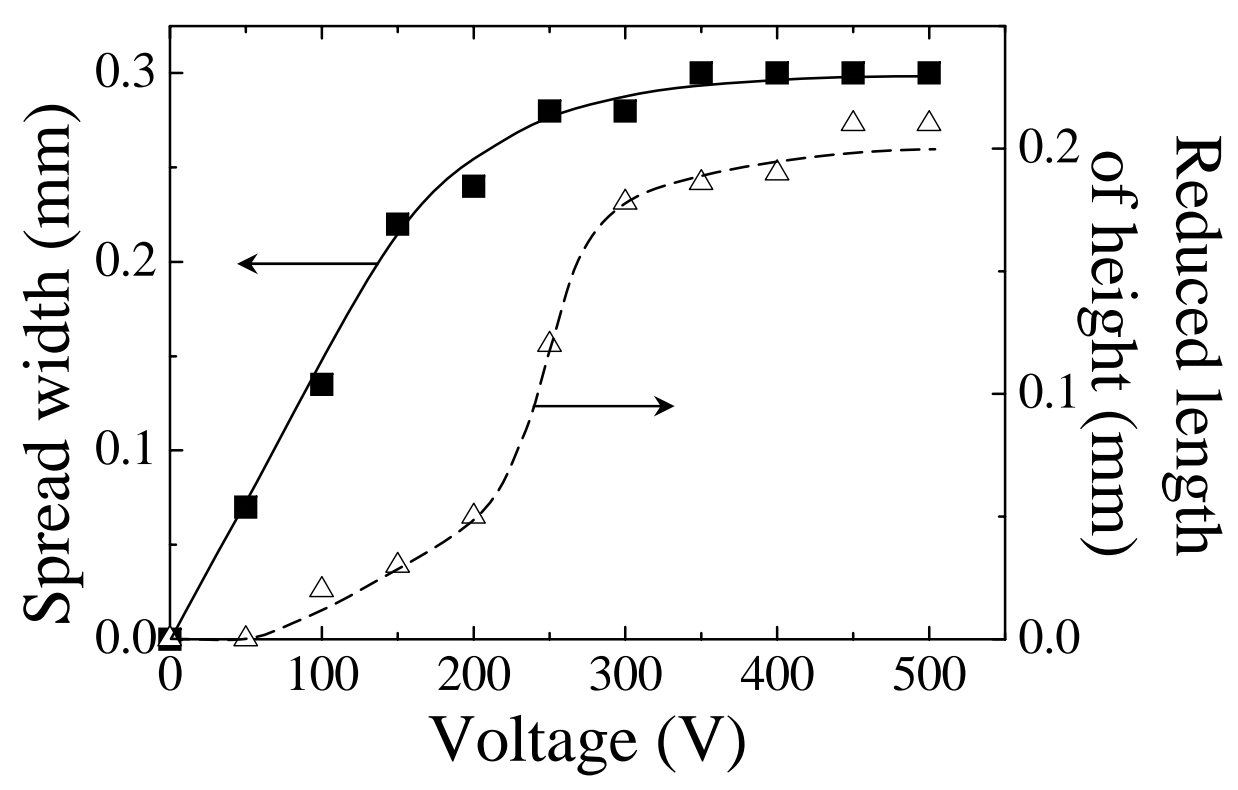

Fig. 2

T. Hino 
(a)

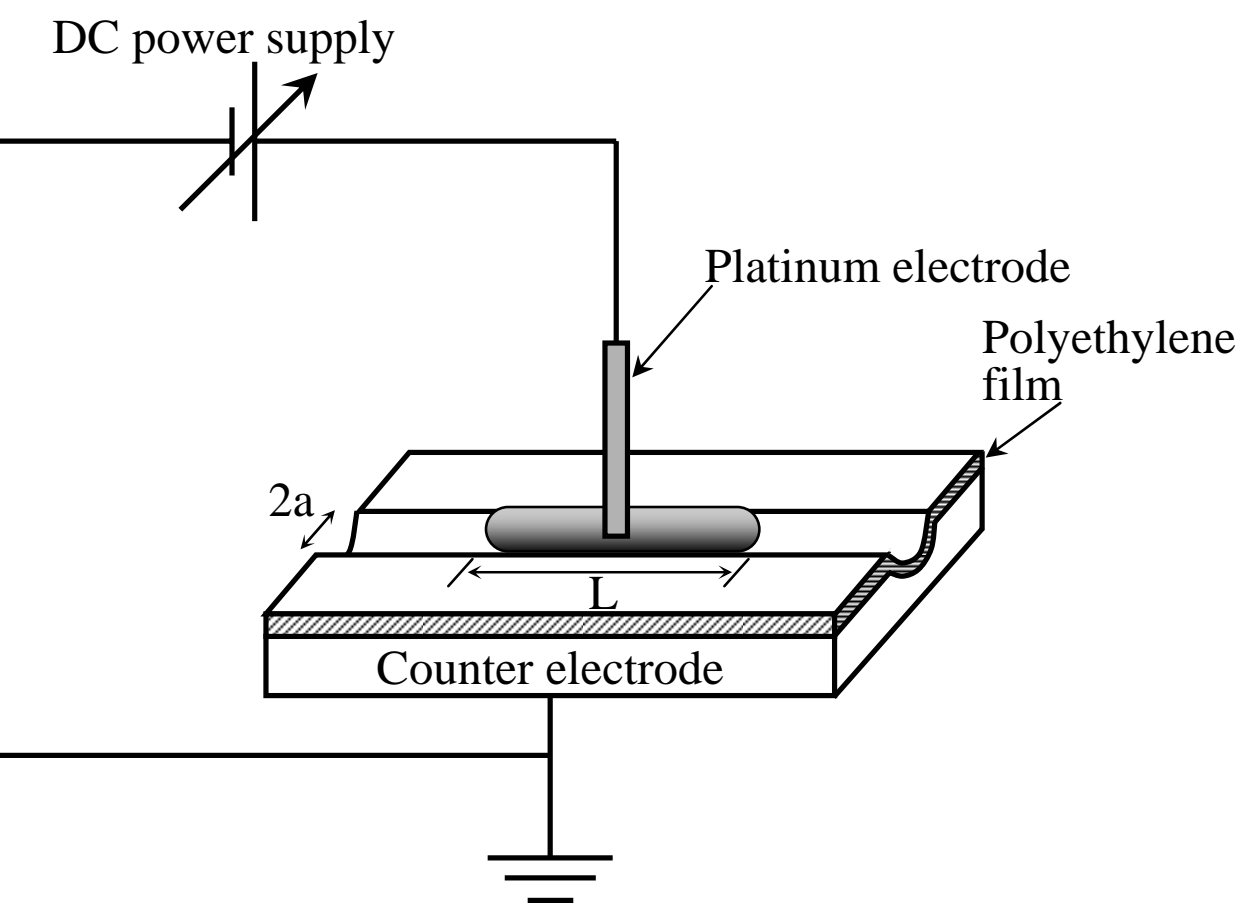

(b)

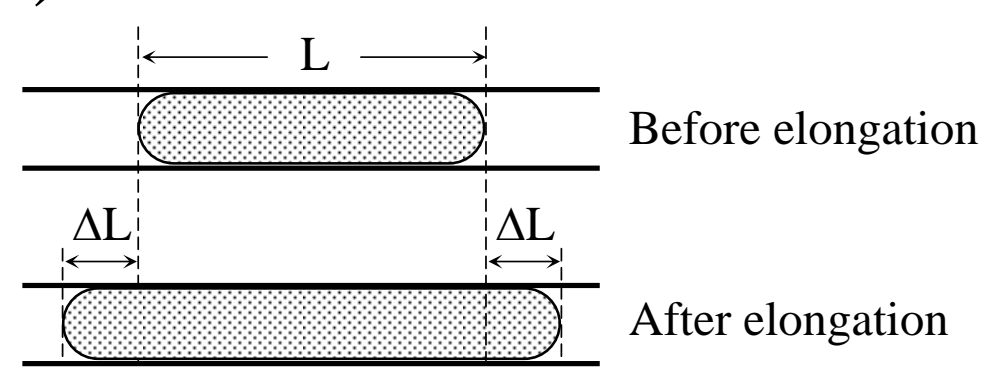

Fig. 3

T. Hino 

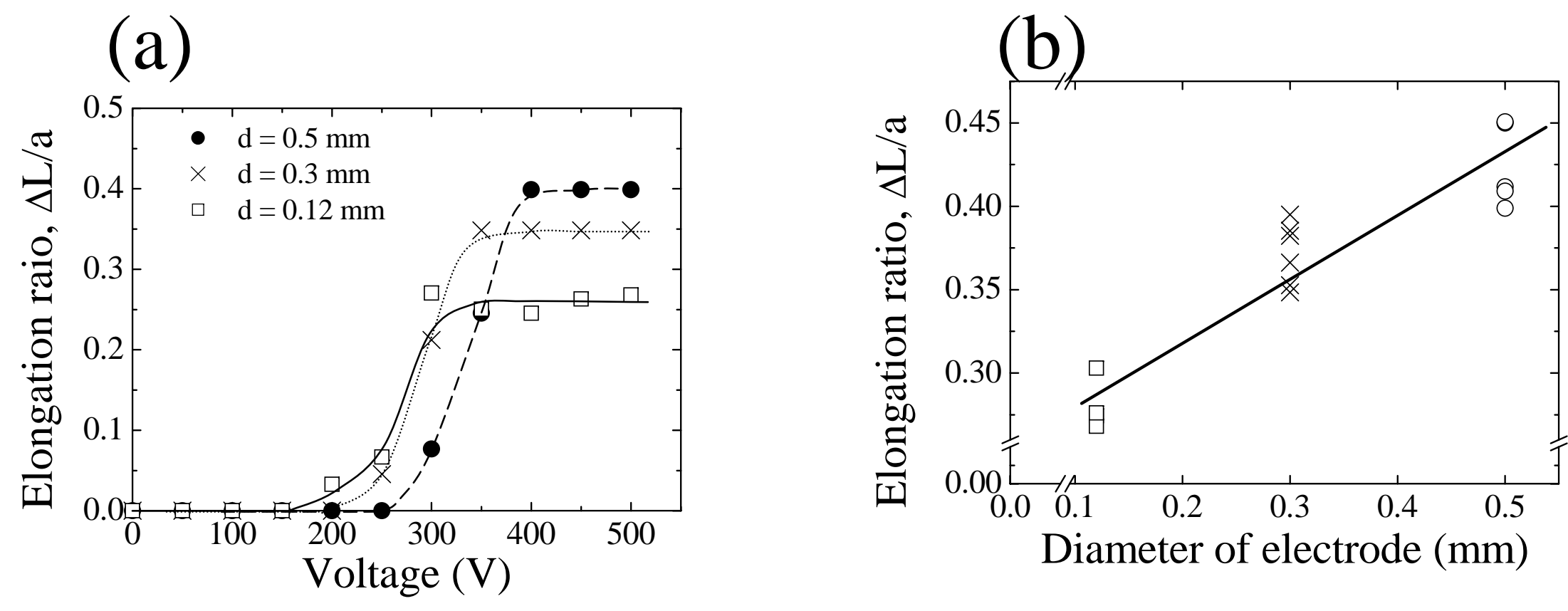

Fig. 4

T. Hino 


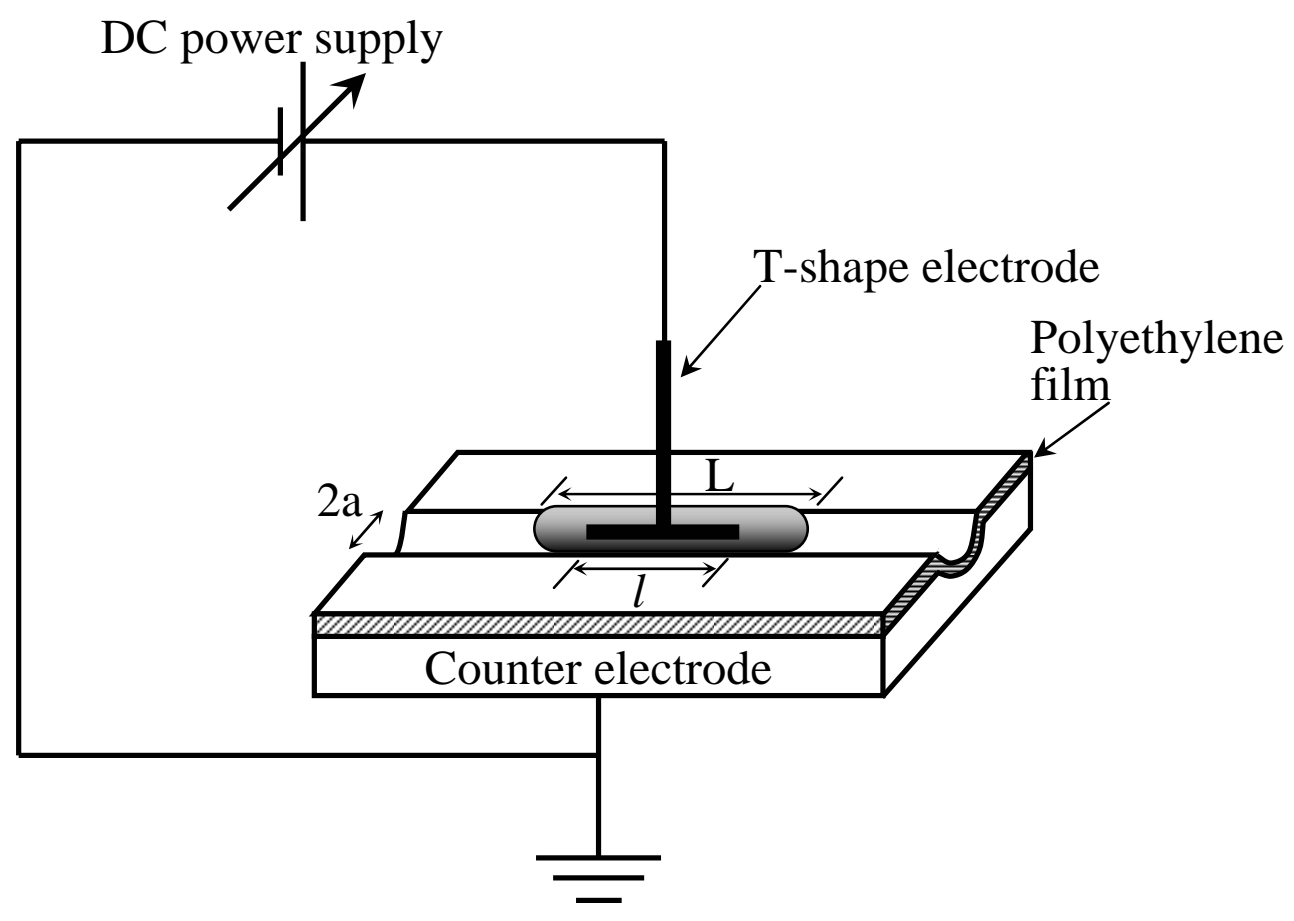

Fig. 5

T. Hino 
(a)

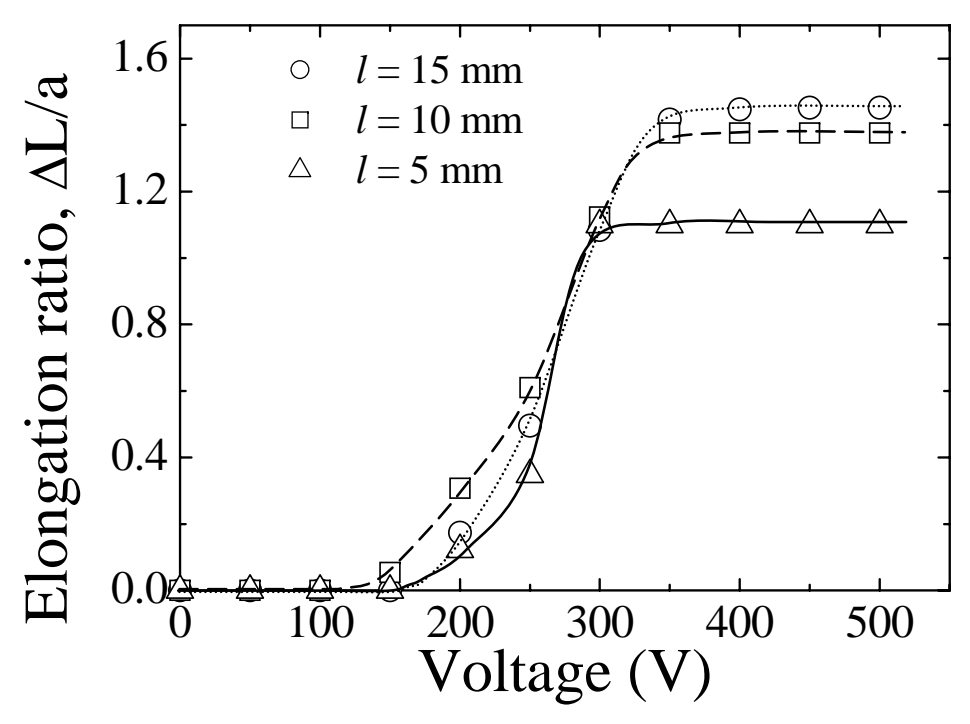

(b)

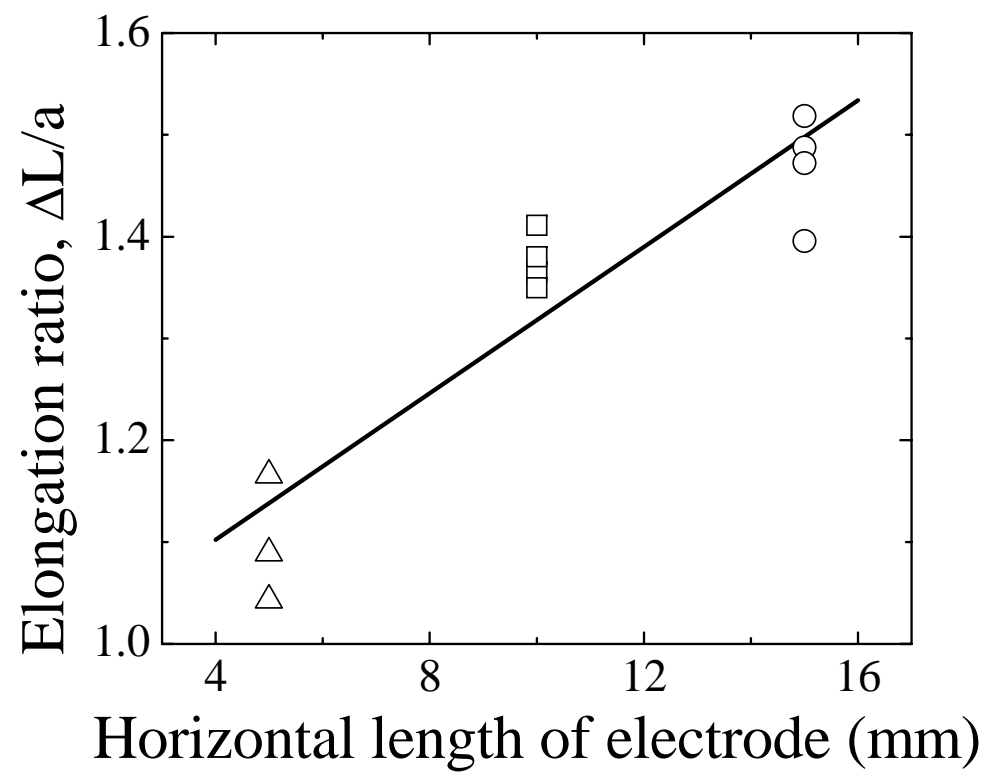

Fig. 6

T. Hino 


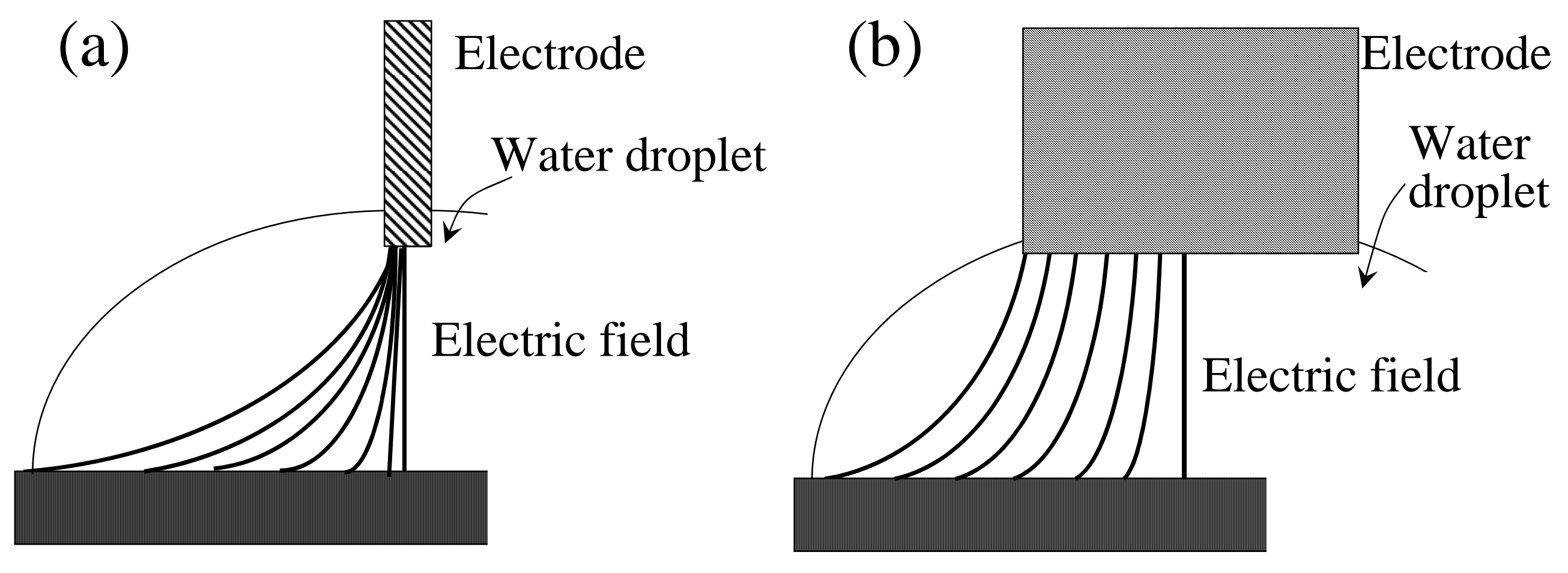

Fig. 7

T. Hino 
(a)

$$
\text { T-shape electrode }
$$

Water droplet

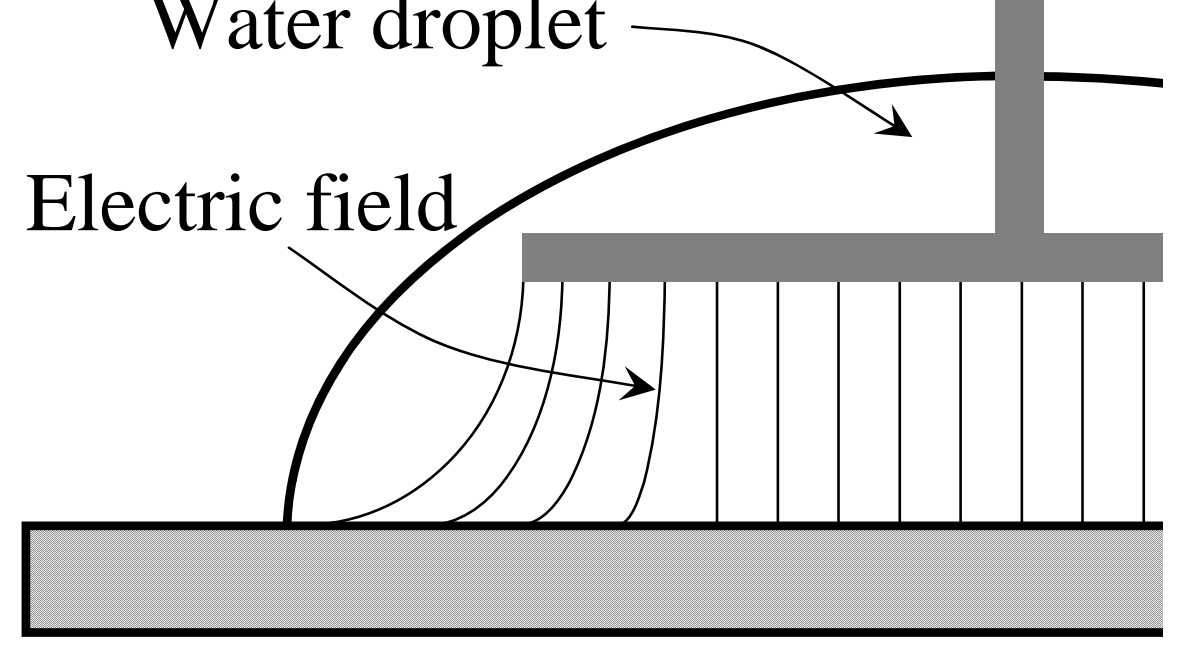

(b)

Fig. 8

T. Hino 